\title{
Composição e riqueza de espécies de anfíbios anuros em três diferentes habitat em um agrossistema no Cerrado do Brasil central
}

\author{
Vitor Azarias Campos ${ }^{1}$, Fabrício Hiroiuki Oda ${ }^{2,6}$, Leandro Juen ${ }^{3}$, Adriane Barth ${ }^{4}$ \& Aline Dartora ${ }^{5}$ \\ ${ }^{1}$ Programa de Pós-graduação em Ecologia e Conservação da Biodiversidade - PPG-ECB, \\ Instituto de Biociências, Universidade Federal de Mato Grosso - UFMT, Av. Fernando Corrêa da Costa, \\ s/n, CCBS-II, Boa Esperança, CEP 78060-900, Cuiabá, MT, Brasil. www.ufmt.br \\ ${ }^{2}$ Núcleo de Pesquisas em Limnologia, Ictiologia e Aqüicultura - Nupélia, Laboratório de \\ Ictioparasitologia, Programa de Pós-graduação em Ecologia de Ambientes Aquáticos Continentais - PEA, \\ Universidade Estadual de Maringá - UEM, Av. Colombo, 5790, Bloco G-90, CEP 87020-900, \\ Maringá, PR, Brasil. http://ictioparasito.nupelia.uem.br/ \\ ${ }^{3}$ Instituto de Ciências Biológicas, Universidade Federal do Pará - UFPA, Rua Augusto Correia, 1, \\ Guama, CEP 66075-110, Belém, PA, Brasil. www.portal.ufpa.br \\ ${ }^{4}$ Programa de Pós-graduação em Genética e Biologia Molecular - PPGGBM, \\ Departamento de Ciências Biológicas, Universidade Estadual de Santa Cruz - UESC, Rod. Ilhéus/Itabuna, \\ Km 16, Salobrinho, CEP 45662-000, Ilhéus, BA, Brasil. www.uesc.br \\ ${ }^{5}$ Laboratório de Zoologia, Departamento de Ciências Biológicas, \\ Universidade do Estado de Mato Grosso - UNEMAT, Campus Universitário de Tangará da Serra, \\ Rod. MT 358, Km 07, Jardim Aeroporto, Tangará da Serra, MT, Brasil. www.tangara.unemat.br \\ ${ }^{6}$ Autor para correspondência: Fabrício Hiroiuki Oda, e-mail: fabricio_oda@hotmail.com
}

CAMPOS, V.A., ODA, F.H., JUEN, L., BARTH, A. \& DARTORA, A. Composition and species richness of anuran amphibians in three different habitat in an agrosystem in Central Brazilian Cerrado. Biota Neotrop. 13(1): http://www.biotaneotropica.org.br/v13n1/en/abstract?inventory+bn03213012013

Abstract: Adaptive plasticity has been postulated as one of the integrating factors that explain the distribution
and abundance of species on different habitat with different environmental variations. The environmental
heterogeneity is the most important factor for the maintenance, increment or decrement of biodiversity. On this
study we determined the effect of periodicity and habitat structure on the richness and composition of species
of three different habitat: stream (P1), temporary ponds (P2) and permanent dam (P3) in an agrosystem in the
Central Brazilian Cerrado. We performed nine excursions to the field during November 2005 to April 2007. We
found and registered the species by direct search and vocalization orientation. We identified 19 anuran species
belonging to four families: Bufonidae (one species), Hylidae (nine species), Leptodactylidae (five species), and
Leiuperidae (four species). The greatest richness and abundance were recorded in temporary ponds (P2), which
differed significantly from the stream (P1) and from the permanent dam (P3). Dendropsophus nanus, Hypsiboas
raniceps and Leptodactylus chaquensis showed strong association with P2. P2 and P3 showed greater differentiation
between themselves in species composition than when compared to P1. Despite the fact that the studied areas
are surrounded by intense farming and present a high degree of anthropic disturbance, these areas showed a high
species richness being an important refugee for the amphibians, although the species found on this work are
usually associated to impacted areas or open phytophysiognomies and are favored with the creation of artificial
environments such as those in this study. Keywords: anurofaunal, adaptative plasticity, environmental heterogeneity, habitat structure, anthropic disturbance.

CAMPOS, V.A., ODA, F.H., JUEN, L., BARTH, A. \& DARTORA, A. Composição e riqueza de espécies de anfíbios anuros em três diferentes habitat em um agrossistema no Cerrado do Brasil central. Biota Neotrop. 13(1): http://www.biotaneotropica.org.br/v13n1/pt/abstract?inventory+bn03213012013

Resumo: A plasticidade adaptativa tem sido postulada como um fator importante para explicar a distribuição e abundância de espécies em habitat com diferentes níveis de variação ambiental e a heterogeneidade ambiental tem sido apontada como responsável pela manutenção, aumento ou diminuição da diversidade. Neste trabalho, determinamos o efeito da periodicidade e estrutura do habitat sobre a riqueza e composição de espécies em três diferentes habitat: córrego $(\mathrm{P} 1)$, poças temporárias $(\mathrm{P} 2)$ e represa permanente $(\mathrm{P} 3)$ em um agrossistema no Cerrado do Brasil central. Nove expedições de campo foram realizadas entre outubro de 2005 e abril de 2007. Os métodos de amostragem por encontro visual e procura auditiva foram utilizados para o registro das espécies. Foram registradas 19 espécies de anuros pertencentes a quatro famílias: Bufonidae (uma espécie), Hylidae (nove espécies), Leptodactylidae (cinco espécies) e Leiuperidae (quatro espécies). Maior riqueza e a abundância foram registradas nas lagoas temporárias (P2), que diferiram significativamente do córrego (P1) e da represa permanente (P3). Dendropsophus nanus, Hypsiboas raniceps e Leptodactylus chaquensis apresentaram forte associação com 
o sítio P2. Os sítios P2 e P3 apresentaram maior diferenciação entre si na composição de espécies, do que quando comparados ao sítio P1. Apesar dos corpos d'água estudados estarem inseridos em área de intensa agricultura e sofrerem elevado grau de perturbação antrópica, esses ambientes apresentam elevada riqueza de espécies, constituindo-se como importantes refúgios para anurofauna da região. Entretanto, as espécies registradas são associadas a áreas antropizadas ou fitofisionomias abertas sendo favorecidas com a criação de ambientes artificiais como os observados no presente estudo.

Palavras-chave: anurofauna, plasticidade adaptativa, heterogeneidade ambiental, estrutura do habitat, perturbação antrópica.

\section{Introdução}

A plasticidade adaptativa tem sido postulada para explicar a distribuição e abundância de espécies em habitat com diferentes níveis de variação ambiental (Griffiths 1997). Existem muitas discussões a respeito dos efeitos que as mudanças na paisagem provocam sobre as comunidades que nela habitam (Knutson et al. 1999, Herrmann et al. 2005, Soares-Filho et al. 2006). As alterações no ambiente podem criar um efeito homogeneizador, levando a uma diminuição na complexidade ambiental e consequentemente na riqueza de espécies (Hazell et al. 2001, Olden et al. 2004, Conte \& Machado 2005, Vasconcelos \& Rossa-Feres 2005).

Considerando que poças são ambientes temporários, os organismos que utilizam esse tipo de habitat devem possuir um desenvolvimento mais rápido, maior amplitude de nicho (mais generalistas) e com maior capacidade de dispersão do que espécies de habitat permanente. Desta forma, seriam menos suscetíveis a variações ambientais, e com as alterações do ambiente, sofreriam menos do que as outras espécies. Com isso poderiam aumentar sua abundância e distribuição, a predominância de tais espécies resultaria em homogeneização biótica e baixa diversidade (veja Griffiths 1997, Leips et al. 2000, Olden et al. 2004).

A heterogeneidade ambiental do Cerrado tem sido utilizada para explicar a riqueza e distribuição local da herpetofauna, visto que o mosaico de habitat contrastantes, abertos e florestais, úmidos e secos (Cavalcanti \& Joly 2002, Oliveira-Filho \& Ratter 2002), distribuídos lado a lado no ambiente, cria um gradiente de condições ambientais e condições variadas de recursos que podem ser exploradas por um grande número de anfíbios e répteis (Brandão \& Araújo 2001, Colli et al. 2002, Nogueira et al. 2009, Oda et al. 2009). Como as espécies possuem exigências eco fisiológicas diferenciadas, os limites de nicho inerentes de cada organismo promovem um gradiente de distribuição das espécies. Segundo Giaretta et al. (2008), a heterogeneidade e descontinuidade dos habitat ajudam a explicar a existência de espécies com distribuição descontinua como por exemplo a Ameerega flavopicta.

Anuros são ectotérmicos, possuem pele permeável e, como conseqüência, são suscetíveis às condições ambientais, que podem afetar sua distribuição e uso de habitat (Duellman \& Trueb 1986). Entretanto, a maioria das espécies, habita regiões que têm altos níveis de umidade e temperaturas moderadas a quentes, devido à permeabilidade de sua pele e da dependência dos habitat aquáticos e terrestres durante seus ciclos de vida (Duellman \& Trueb 1994, Wells 2007). Assim, impactos humanos no ambiente fazem dos anfíbios um dos grupos animais mais vulneráveis a modificações.

Devido à elevada dependência que anuros têm da qualidade ambiental, tem sido demonstrado em algumas áreas que fatores abióticos (chuva, temperatura e heterogeneidade da vegetação) têm maiores efeitos sobre a estrutura das comunidades de anuros do que os fatores bióticos (competição e predação) (Vasconcelos et al. 2009). Assim, acreditamos que a heterogeneidade ambiental e hidroperíodo instável de habitat perenes influenciam fortemente a riqueza e composição de espécies do que habitat permanentes. Dessa forma, o objetivo deste trabalho foi determinar o efeito da periodicidade $\mathrm{e}$ estrutura do habitat sobre a riqueza e composição de espécies em três diferentes habitat em um agrossistema no Cerrado do Brasil central.

\section{Material e Métodos}

\section{1. Área de estudo}

O estudo foi realizado em três tipos de corpos d'água dentro do domínio fitogeográfico do Cerrado, localizados no município de Tangará da Serra (Figura 1), situados a 6,5 km a oeste da cidade de

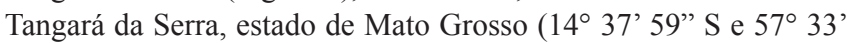
39 ” O). A região situa-se a $240 \mathrm{~km}$ a noroeste da capital Cuiabá, localizada entre as serras de Tapirapuã e dos Parecis, apresentando relevo de topografia plana (Soares \& Nogueira 2008).

A precipitação anual varia entre 1300 a $2000 \mathrm{~mm}$ com um período de chuvas (setembro-abril) e de seca (maio-agosto) bem determinados. A temperatura oscila entre 16 e $36{ }^{\circ} \mathrm{C}$, com umidade relativa média de $80 \%$ (Cruz et al. 2008). Apesar da ocorrência de Floresta Estacional Semidecidual, a vegetação da região é predominantemente caracterizada pela presença de formações savânicas e áreas antrópicas (Brasil 1982). As principais atividades econômicas são pecuária de corte, cultivo de soja, algodão, café, arroz e cana-de-açúcar.

Foram amostrados três tipos de ambiente, córrego no interior de floresta de galeria $\left(14^{\circ} 37^{\prime} 57^{\prime}\right.$ 'S e $57^{\circ} 33$ ' 38” O) (Sítio P1), lagoas temporárias na borda de floresta de galeria $\left(14^{\circ} 37^{\prime} 58^{\prime \prime} \mathrm{S}\right.$ e $57^{\circ} 33^{\prime}$ 39” O) (Sítio P2) e represa permanente (14 37' 59" S e 57 33'36” O) (Sítio P3). Sítio P1 (148,48 m²): a vegetação é constituída por árvores de médio a grande porte e palmeiras de Bacuri (Platonia insignis Mart.). O solo apresenta grande quantidade de serapilheira e rochas. A água possui aspecto cristalino, com fundo formado por rochas e areia. No Sítio P2 $\left(184,90 \mathrm{~m}^{2}\right)$ : para que o tamanho amostral fosse similar a área amostrada nos outros tratamentos foi considerado as três lagoas como sendo uma única área. A distância entre as lagoas era de aproximadamente $2 \mathrm{~m}$. A margem em contato com a floresta de galeria é composta por árvores de médio a grande porte, cobertas por lianas herbáceas e lenhosas. As outras margens são constituídas por capim navalha (Panicum maximum Jacq.), arbustos e Embaúba (Cecropia sp.). Durante o período da seca o interior das lagoas apresenta o solo úmido com predomínio de $P$. maximum. A água possui aspecto cristalino, com substrato formado de matéria orgânica vegetal em decomposição. O Sítio P3 $\left(171,49 \mathrm{~m}^{2}\right)$ : é formado a partir do represamento do córrego e é utilizada para criação de peixes. A vegetação marginal é constituída por gramíneas, arbustos, árvores de médio a grande porte e palmeiras de Bacuri ( $P$. insignis). A água possui aspecto túrbido, com substrato formado por barro. 


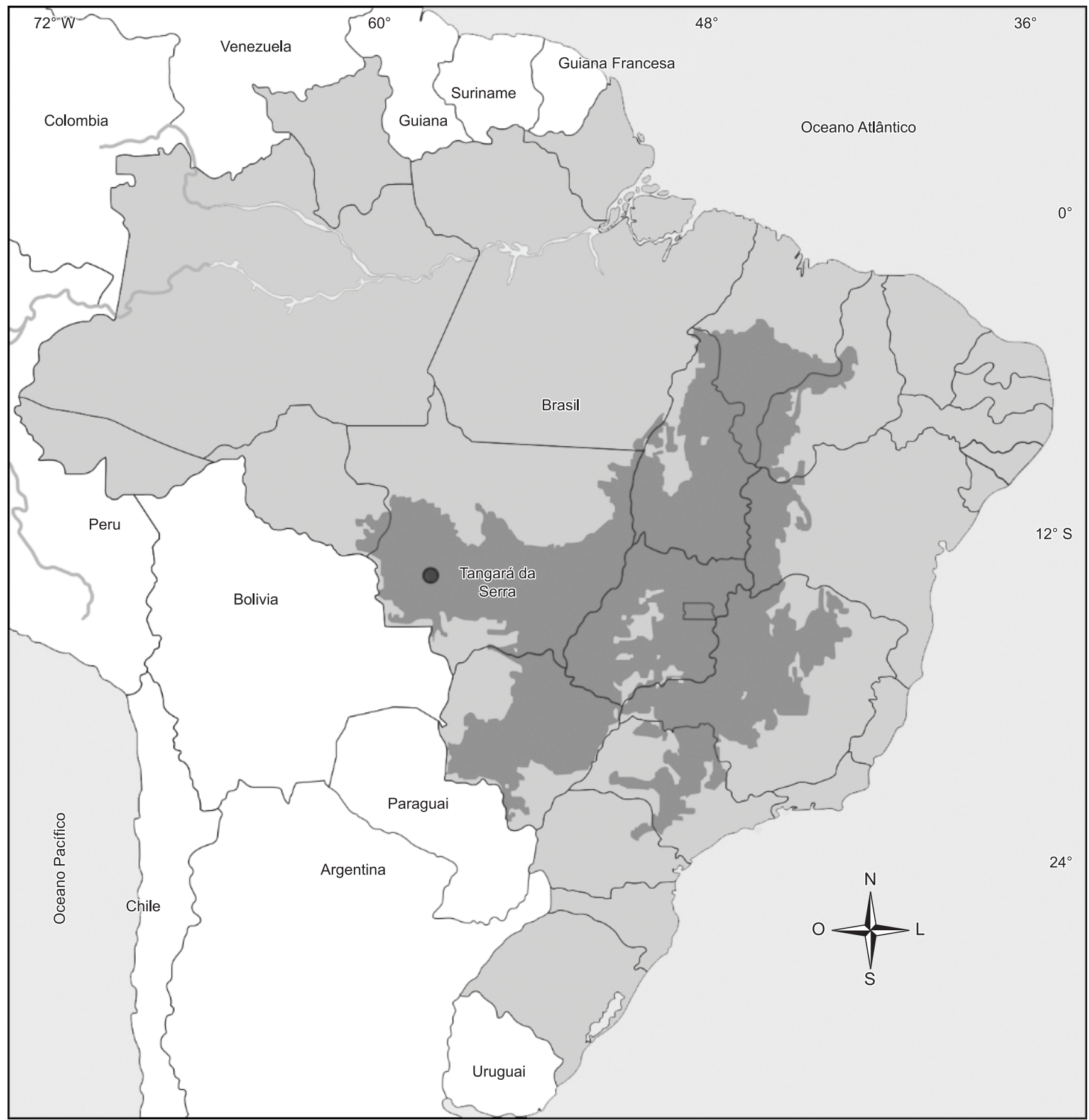

Figura 1. Localização do município de Tangará da Serra no estado de Mato Grosso, Brasil. O domínio fitogeográfico do Cerrado é mostrado em cinza escuro.

Figure 1. Location of the municipality of Tangará da Serra in the state of Mato Grosso, Brazil. The Cerrado phytogeographic domain is displayed in dark gray.

\section{Amostragem}

Nove expedições de campo foram realizadas na área de estudo entre outubro de 2005 e abril de 2007. As amostragens foram feitas pelos métodos de encontro visual (Crump \& Scott Junior 1994) e procura auditiva (Zimmerman 1994), durante o período noturno em cada corpo d'água.

Alguns indivíduos de cada espécie foram coletados, fixados em solução de formalina a $10 \%$ e posteriormente conservados em etanol a 70\%. Exemplares-testemunho foram depositados no Museu de Zoologia de Tangará da Serra (MZT) - Coleção Herpetológica da
Universidade Estadual de Mato Grosso. Licença de coleta concedida pelo Instituto Brasileiro do Meio Ambiente e Recursos Naturais Renováveis (IBAMA; permissão: 17914-2, processo: 21619-1).

\section{Análises estatísticas}

Considerando que a riqueza de espécies observada é freqüentemente um estimador viciado em relação à riqueza de espécies real (Santos 2006), foi usado o estimador não paramétrico Jackknife de $1^{\mathrm{a}}$ ordem para extrapolação da riqueza de espécies na área de estudo. Este estimador atribui maior peso às espécies raras (aquelas que ocorreram em apenas uma amostra) (Burnham 
\& Overton 1979). Mesmo que o método subestime a riqueza da comunidade, isto não constitui um grande problema, uma vez que em geral o que se espera das estimativas de riqueza é obter parâmetros para comparações entre inventários (Santos 2004). Para um melhor suporte dos resultados, também foi realizada a rarefação padronizada por amostra, pois conforme argumenta Courtemanch (1996), não se pode comparar amostras padronizadas por uma certa quantidade de indivíduos, visto que se perde muita informação quanto à abundância/ densidade das espécies nas comunidades. A técnica de rarefação utilizada foi a "baseada em momentos" (Mao Tau) (Gotelli \& Colwell 2001), que elimina completamente a necessidade de réplica e permite a comparação direta de riqueza entre amostras (Colwell et al. 2004). Ambas as técnicas de estimativas foram calculadas no programa EstimateS 7.5.0 (Colwell 2005).

Para testar se houve diferenças na abundância de indivíduos entre as três áreas, aplicou-se a análise de variância (ANOVA unifatorial) (Zar 1999). Os pressupostos de normalidade e de homocedasticidade (teste de Levene) foram calculados e testados. Quando os pressupostos não foram satisfatórios, a abundância foi transformada usando $\log _{10}+1$. Quando a $A N O V A$ foi significativa, aplicou-se o teste de Tukey para identificar quais locais apresentaram médias diferentes.

Para verificar a associação das espécies a um determinado local usamos o Teste de Espécies Indicadoras (IndVal) de Dufrêne \& Legendre (1997), submetidos ao teste randomização de Monte Carlo, para os três locais de amostragem. Este teste analisa a fidelidade e a especificidade das espécies a uma determinada variável, servindo para mensurar o grau de associação.

Para sumarizar os dados da estrutura e composição da assembléia, utilizou-se a análise de correspondência com remoção do efeito do arco
(ACD) (Hill \& Gauch 1980, Gauch 1982, Ter Braak 1995) ordenando os locais estudados com base na similaridade de composição a partir de dados de abundância das espécies transformados $(\log +1)$. Para testar a diferença na composição de espécies entre as áreas, aplicou-se a Análise de Similaridade Bi-fatorial (ANOSIM), método usado para detectar diferenças na estrutura da composição (Melo \& Hepp 2008).

\section{Resultados}

Foram registradas 19 espécies de anuros pertencentes a quatro famílias: Bufonidae (uma espécie), Hylidae (nove espécies), Leptodactylidae (cinco espécies) e Leiuperidae (quatro espécies, Tabela 1). Maior riqueza foi observada no sítio P2 com 16 espécies, seguido pelos sítios P3 e P1 com 11 e 10 espécies, respectivamente (Tabela 1).

As curvas de rarefação de espécies baseadas em esforço de coleta nos três sítios reprodutivos apresentaram forte tendência à estabilização (Figura 2), representando que o esforço amostral foi suficiente para registrar as espécies da localidade. Usando os dados gerados pelo estimador Jackknife e a técnica de inferência por intervalo de confiança, o sítio $\mathrm{P} 2$ teve maior riqueza de espécies $22,81 \pm 4.96$ (média \pm intervalo de confiança), apresentando em média nove espécies a mais do que P3 $(13,88 \pm 1,60)$, mas não apresentou diferença significativa com $\mathrm{P} 1(16,73 \pm 8,36)$, pois houve sobreposição do intervalo de confiança de um grupo com a média do outro. O mesmo foi observado quando foram comparados os sítios P3 e P1 (Figura 3).

O padrão de abundância das espécies diferiu entre os sítios reprodutivos amostrados (Tabela 1). No sítio P1, foram registrados

Tabela 1. Espécies e abundância de anuros registradas em três ambientes no município de Tangará da Serra, estado de Mato Grosso, Brasil.

Table 1. Species and abundance of anurans registered in three environments in the municipality of Tangará da Serra, Mato Grosso state, Brazil.

\begin{tabular}{|c|c|c|c|c|c|c|c|}
\hline \multirow{3}{*}{ Famílias e espécies } & \multirow{3}{*}{$\mathbf{N}$} & \multicolumn{6}{|c|}{ Ambientes amostrados } \\
\hline & & \multicolumn{2}{|c|}{ P1 } & \multicolumn{2}{|c|}{$\mathbf{P 2}$} & \multicolumn{2}{|c|}{ P3 } \\
\hline & & N1 & $\%$ & $\mathbf{N 2}$ & $\%$ & N3 & $\%$ \\
\hline \multicolumn{8}{|l|}{ BUFONIDAE } \\
\hline Rhinella schneideri (Werner, 1894) & 32 & 2 & 12,5 & 28 & 3,0 & 2 & 1,8 \\
\hline \multicolumn{8}{|l|}{ HYLIDAE } \\
\hline Dendropsophus melanargyreus (Cope, 1887) & 2 & - & - & 2 & 0,2 & - & - \\
\hline Dendropsophus minutus (Peters, 1872) & 5 & 1 & 6,2 & 3 & 0,3 & 1 & 0,9 \\
\hline Dendropsophus nanus (Boulenger, 1889) & 379 & 1 & 6,2 & 366 & 39,8 & 12 & 10,9 \\
\hline Hypsiboas geographicus (Spix, 1824) & 139 & 1 & 6,2 & 107 & 11,6 & 31 & 28,1 \\
\hline Hypsiboas punctatus (Schneider, 1799) & 63 & 1 & 6,2 & 23 & 2,5 & 39 & 35,4 \\
\hline Hypsiboas raniceps Cope, 1862 & 54 & 3 & 18,7 & 41 & 4,5 & 10 & 9,1 \\
\hline Scinax fuscomarginatus (A. Lutz, 1925) & 280 & 4 & 25,0 & 275 & 29,9 & 1 & 0,9 \\
\hline Scinax fuscovarius (A. Lutz, 1925) & 1 & - & - & 1 & 0,1 & - & - \\
\hline Scinax nebulosus (Spix, 1824) & 32 & - & - & 28 & 3,0 & 4 & 3,6 \\
\hline \multicolumn{8}{|l|}{ LEIUPERIDAE } \\
\hline Eupemphix nattereri Steindachner, 1863 & 3 & - & - & - & - & 3 & 2,7 \\
\hline Physalaemus albonotatus (Steindachner, 1864) & 7 & 1 & 6,2 & 6 & 0,6 & - & - \\
\hline Physalaemus centralis Bokermann, 1962 & 5 & - & - & 5 & 0,5 & - & - \\
\hline Physalaemus cuvieri Fitzinger, 1826 & 3 & - & & 3 & 0,3 & - & - \\
\hline \multicolumn{8}{|l|}{ LEPTODACTYLIDAE } \\
\hline Leptodactylus chaquensis Cei, 1950 & 22 & - & - & 18 & 2,0 & 4 & 3,6 \\
\hline Leptodactylus fuscus (Schneider, 1799) & 6 & - & & 6 & 0,6 & - & - \\
\hline Leptodactylus labyrinthicus (Spix, 1824) & 2 & 1 & 6,2 & 1 & 0,1 & - & - \\
\hline Leptodactylus mystacinus (Burmeister, 1861) & 2 & 1 & 6,2 & 1 & 0,1 & - & - \\
\hline Leptodactylus podicipinus (Cope, 1862) & 7 & - & - & 4 & 0,4 & 3 & 2,7 \\
\hline Número de indivíduos & 1044 & 16 & & 918 & & 110 & \\
\hline Número de espécies & 19 & 10 & & 18 & & 11 & \\
\hline
\end{tabular}



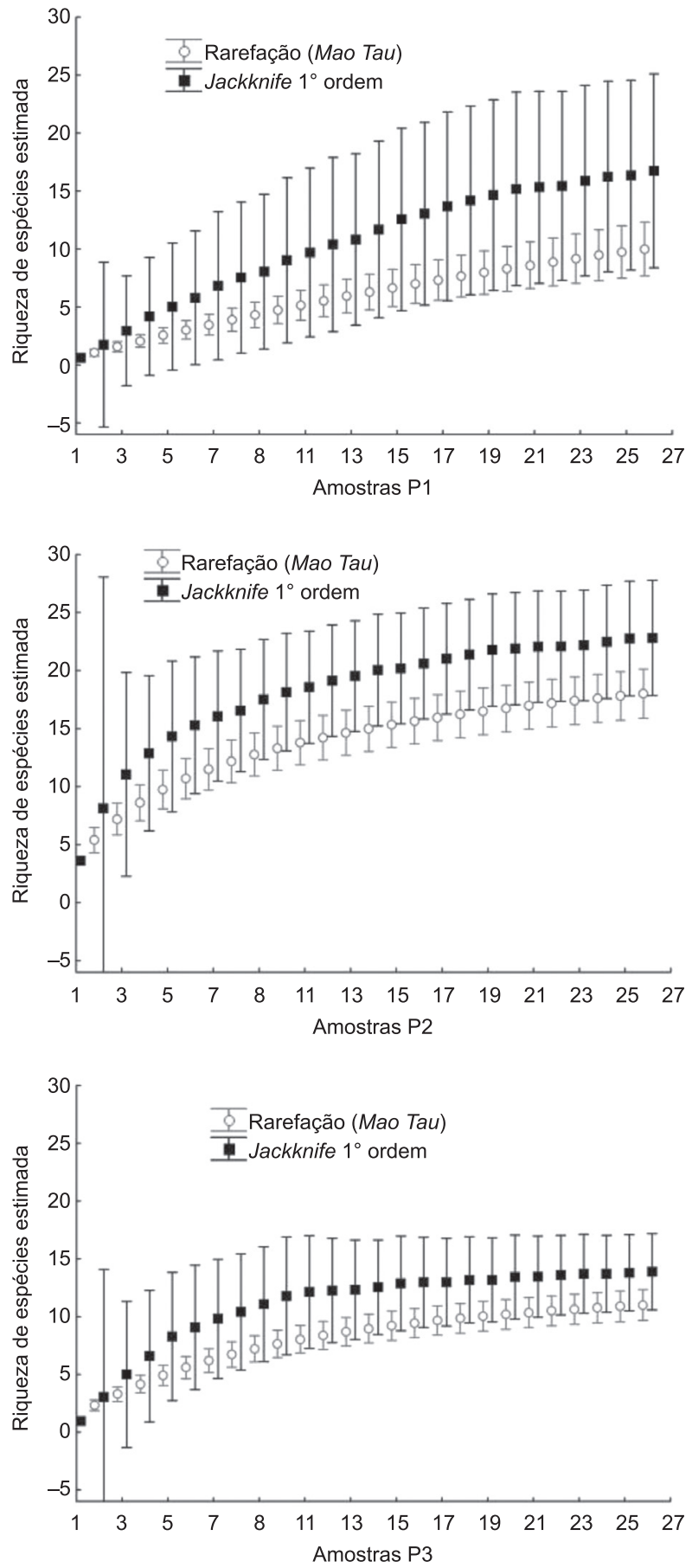

Figura 2. Curvas de rarefação de espécies de anfíbios anuros geradas pelo estimador Jackknife 1 em três ambientes no município de Tangará da Serra, estado de Mato Grosso, Brasil. a) córrego no interior de floresta de galeria, b) lagoas temporárias na borda de floresta de galeria e c) represa permanente.

Figure 2. Rarefaction curves of amphibian anuran species generated by the estimator Jackknife 1 in three environments in the municipality of Tangará da Serra, Mato Grosso state, Brazil. a) stream inside gallery forest, b) temporary ponds in the edge of gallery forest, and c) permanent dam.

apenas 16 indivíduos. No sítio $\mathrm{P} 2$, foram registrados 918 indivíduos. No sítio P3, foram registrados 110 indivíduos (Tabela 1). Diferença significativa foi observada na abundância do sítio P2 em relação aos sítios P1 e P3 $\left(\mathrm{F}_{(2,36)}=15,884 ; \mathrm{p}<0,001\right)$. O sítio P2 apresentou em média 65 indivíduos a mais do que os sítios P1 e P3. Não foi

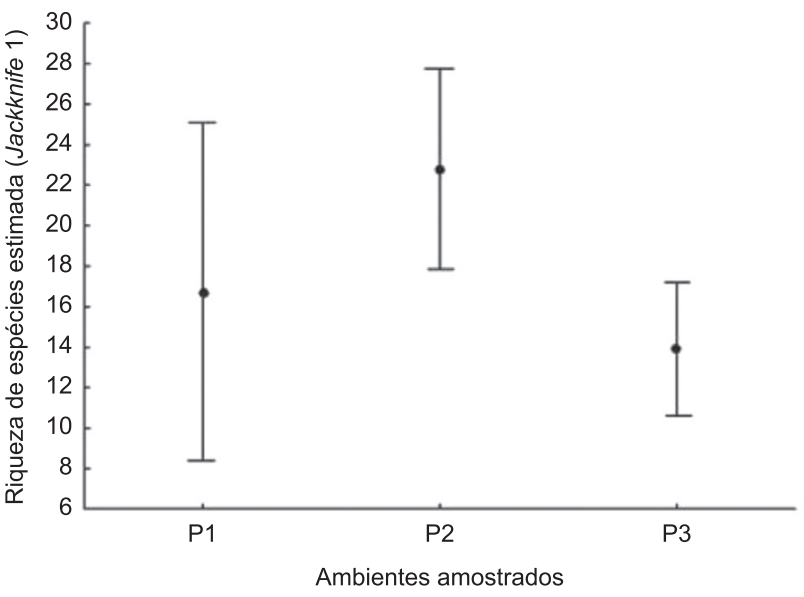

Figura 3. Riqueza estimada (estimador Jackknife 1) de espécies de anfíbios anuros em três ambientes no município de Tangará da Serra, estado de Mato Grosso, Brasil. P1: córrego no interior de floresta de galeria, P2: lagoas temporárias na borda de floresta de galeria e P3: represa permanente.

Figure 3. Estimated species richness (estimador Jackknife 1) of amphibian anuran species in three environments in the municipality of Tangará da Serra, Mato Grosso state, Brazil. P1: stream inside gallery forest, P2: temporary ponds in the edge of gallery forest, and P3: permanent dam.

observada diferença significativa na abundância entre os sítios P1 e P3 (Figura 4).

Dentre as 19 espécies registradas no estudo, 95\% ocorreram no sítio P2 (lagoas temporárias). Cerca de 37\% das espécies ocorreram nos três sítios (Rhinella schneideri, Dendropsophus minutus, D. nanus, Hypsiboas geographicus, H. punctatus, H. raniceps, Scinax fuscomarginatus), 32\% ocorreram em dois sítios (Physalaemus albonotatus, Leptodactylus chaquensis, L. labyrinthicus, L. mystacinus, L. podicipinus, Scinax nebulosus) e 32\% ocorreram em apenas um dos sítios (Eupemphix nattereri, Physalaemus centralis, P. cuvieri, Leptodactylus fuscus, Dendropsophus melanargyreus, Scinax fuscovarius). Por meio do Teste de Espécies Indicadoras (IndVal) foi observado que Dendropsophus nanus, Hypsiboas raniceps e Leptodactylus chaquensis apresentaram forte associação com o sítio P2 (Tabela 2).

Através da ordenação das amostras dos sítios reprodutivos produzida pela ACD foi possível constatar que os sítios P2 e P3 apresentaram maior diferenciação entre si na composição de espécies, do que quando comparados ao sítio P1 (Figura 5). Este resultado é confirmado pela análise $A N O S I M$ onde foi observada diferença significativa entre os sítios P2 e P3 $(R=0,114 ; p=0,042)$. Por outro lado, não foram encontradas diferenças significativas entre os sítios P1 e P2 $(R=0,060 ; p=0,300)$ e P1 e P3 $(R=-0,009 ; p=0,479)$.

\section{Discussão}

A taxocenose de anfíbios anuros da área estudada é composta em sua maioria (79\%) por espécies amplamente distribuídas na América do Sul, freqüentemente associadas a áreas antropizadas ou fitofisionomias abertas (Rhinella schneideri, Dendropsophus melanargyreus, D. minutus, D. nanus, Hypsiboas geographicus, H. punctatus, $H$. raniceps, Scinax fuscomarginatus, $S$. fuscovarius, $S$. nebulosus, Physalaemus cuvieri, Leptodactylus fuscus, L. labyrinthicus, L. mystacinus e L. podicipinus) (Bernarde \& Kokubum 1999, Vasconcelos \& Rossa-Feres 2005, Santos et al. 2007, 2009). Apenas $21 \%$ das espécies estão relacionadas às formações abertas do Chaco (Physalaemus albonotatus e Leptodactylus chaquensis) e Cerrado (Eupemphix nattereri e Physalaemus centralis). 
Tabela 2. Valor individual de indicação (IndVal) das espécies de anuros amostradas em três ambientes no município de Tangará da Serra, estado de Mato Grosso, Brazil.

Table 2. Indication individual value (IndVal) of the anuran species sampled in the three environments in the municipality of Tangará da Serra, Mato Grosso state, Brazil.

\begin{tabular}{lccc}
\hline \multicolumn{1}{c}{ Espécies } & Valor da indicação (IndVal) & p & Ambiente \\
\hline Dendropsophus nanus & 58,9 & 0,028 & P2 \\
Hypsiboas raniceps & 52,1 & 0,138 & P2 \\
Leptodactylus chaquensis & 49,6 & 0,066 & P2 \\
Hypsiboas geographicus & 40,1 & 0,218 & P2 \\
Scinax fuscomarginatus & 36,6 & 0,164 & $\mathrm{P} 2$ \\
Scinax nebulosus & 33,5 & 0,108 & $\mathrm{P} 3$ \\
Hypsiboas punctatus & 30,8 & 0,303 & $\mathrm{P} 3$ \\
Eupemphix nattereri & 25 & 0,107 & $\mathrm{P} 3$ \\
Rhinella schneideri & 23,5 & 0,472 & $\mathrm{P} 2$ \\
Leptodactylus fuscus & 23,1 & 0,179 & $\mathrm{P} 2$ \\
Leptodactylus labyrinthicus & 19,1 & 0,142 & $\mathrm{P} 1$ \\
Leptodactylus mystacinus & 19,1 & 0,142 & $\mathrm{P} 1$ \\
Dendropsophus minutus & 15,5 & 0,264 & $\mathrm{P} 1$ \\
Physalaemus centralis & 15,4 & 0,610 & $\mathrm{P} 2$ \\
Physalaemus albonotatus & 15 & 0,466 & $\mathrm{P} 2$ \\
Leptodactylus podicipinus & 12,7 & 0,749 & $\mathrm{P} 2$ \\
Dendropsophus melanargyreus & 7,7 & 1,000 & $\mathrm{P} 3$ \\
Scinax fuscovarius & 7,7 & 1,000 & $\mathrm{P} 2$ \\
Physalaemus cuvieri & 6,9 & 0,878 & \\
\hline
\end{tabular}

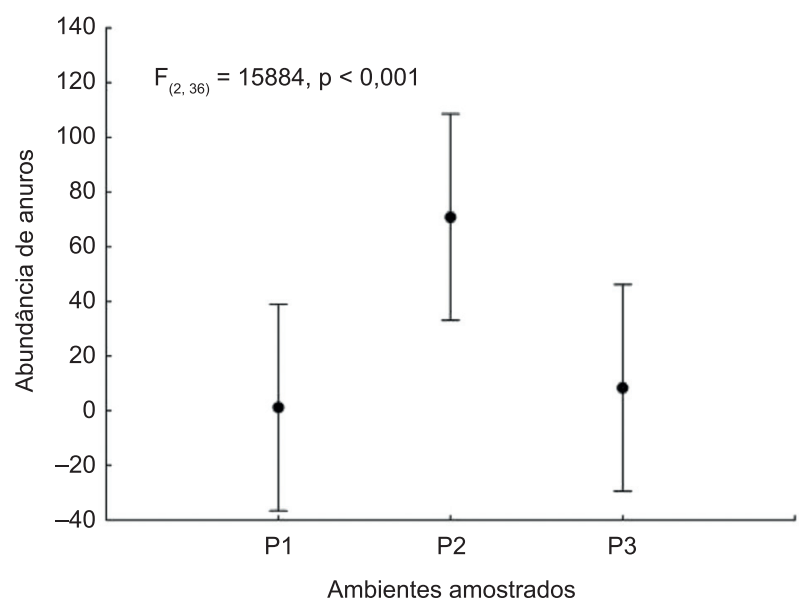

Figura 4. Abundância das espécies de anfíbios anuros registradas em três ambientes no município de Tangará da Serra, estado de Mato Grosso, Brasil.

Figure 4. Abundance of amphibian anuran species registered in three environments in the municipality of Tangará da Serra, Mato Grosso state, Brazil.

A forte tendência a estabilização das curvas de rarefação, bem como a riqueza estimada para os três sítios reprodutivos analisados, mostraram que o método de coleta empregado foi apropriado para a determinação da riqueza local, uma vez que a eficiência de captura foi de $60 \%$ para P1 (riqueza observada/observada), 79\% para P2 e $80 \%$ para $\mathrm{P} 3$. Estudos recentes como os realizados no Alto Tocantins, Niquelândia, Goiás (29 espécies; Oda et al. 2009), região sudoeste de Goiás (36 espécies; Morais et al. 2011) e que usaram a mesma

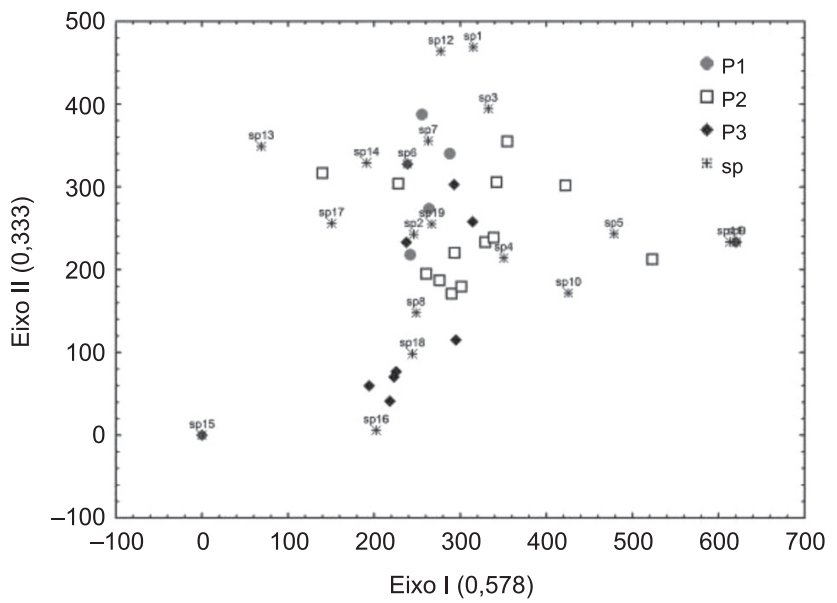

Figura 5. Diagrama de ordenação da Análise de Correspondência Destendenciada (ACD) baseado na composição de espécies de anfíbios anuros em três ambientes no município de Tangará da Serra, estado de Mato Grosso, Brasil.

Figure 5. Ordination diagram of Detrented Correspondence Analysis $(D C A)$ based in the composition of amphibian anuran species in three environments in the municipality of Tangará da Serra, Mato Grosso state, Brazil.

metodologia também obtiveram resultados similares, resultando a eficiência amostral do nosso estudo.

Maior número de espécies e abundância foram encontrados em corpos d'água temporários (lagoas temporárias na borda de floresta de galeria), corroborando resultados obtidos em outros estudos em regiões de clima sazonal (e.g. Prado et al. 2005, Santos et al. 2007, Vasconcelos et al. 2009), onde a maioria das espécies também utilizou corpos d'água temporários para reprodução. Williams et al. (2003) 
também evidenciaram uma elevada biodiversidade em lagoas quando comparadas a rios, riachos e diques em área de agricultura.

Em nosso estudo, a não utilização de réplicas espaciais é justificada pela dificuldade de instituí-las de forma que se represente fielmente os corpos d'água com os mesmos tipos de impactos, uma vez que a variação na composição das espécies pode existir por qualquer outro fator externo desconhecido, o que poderia mascarar nossos resultados. Assim, a elevada riqueza em corpos d'água temporários quando comparados com a lagoa permanente e o córrego, concordam com os resultados obtidos em outros estudos em regiões de clima sazonal (e.g. Prado et al. 2005, Santos et al. 2007, Vasconcelos et al. 2009), onde a maioria das espécies utilizou poças temporárias para reprodução. Além disso, os ambientes temporários, onde os predadores de girinos são menos abundantes ou ausentes, podem atrair maior número de espécies de anuros que os ambientes permanentes (Smith 1983, Woodward 1983, Skelly 1997), permitindo as larvas explorarem fontes de alimento antes da colonização de insetos e peixes, enquanto ao mesmo tempo, evitam predadores (Barreto \& Moreira 1996, Eterovick \& Sazima 2000, Prado et al. 2005). Entretanto, Kopp \& Eterovick (2006) observaram que a riqueza de espécies de girinos não foi associada ao número de tipos ou abundância de potenciais predadores de girinos em poças, sugerindo que fatores ambientais e estocásticos são mais prováveis a produzir os padrões observados de distribuição das espécies do que as interações bióticas.

Neste estudo, dois fatores não mutuamente exclusivos podem explicar a maior riqueza e composição de espécies em P2 (três lagoas temporárias na borda de floresta de galeria): 1) a heterogeneidade estrutural do habitat e 2) a plasticidade no desenvolvimento larval das espécies de anuros. O diversificado estrato vegetal (arbóreas, arbustivas e herbáceas) presente nesse ambiente possibilita a ocupação vertical do habitat pelos hilídeos que juntamente com as espécies terrestres, principalmente leiuperídeos e leptodactilídeos, contribuem para a maior diversificação e número de espécies nesse ambiente (veja Cardoso et al. 1989, Pombal 1997).

Já a plasticidade no desenvolvimento larval permite aos anuros ajustarem as taxas reprodutivas de acordo com a variação ambiental do habitat (veja Griffiths 1997). Como exemplo, anuros quando expostos a condições favoráveis (baixo risco de predação e de dessecação do habitat) apresentam um maior período larval, o qual garante tamanho de corpo maior durante a metamorfose, aprimora o sucesso de acasalamento dos machos, aumenta a probabilidade de sobrevivência durante a hibernação, diminui o tempo para a primeira reprodução e aumenta a fecundidade das fêmeas (Howard 1978, Berven 1981, 1982, 1990, Berven \& Gill 1983, Pough \& Kamel 1984, Smith 1987, Semlitsch et al. 1988). Por outro lado, em condições menos favoráveis (alto risco de predação e dessecação), os anuros diminuem o período larval o qual minimiza o risco acumulado de mortalidade por predação ou dessecação do habitat, mas com um custo de menor tamanho do corpo durante a metamorfose e suas desvantagens concomitantes (Leips et al. 2000). Desta forma, mesmo com eventos catastróficos de extinção em uma lagoa, a imigração de juvenis e adultos de lagoas vizinhas pode assegurar a continuidade da população (Griffiths 1997). Sendo assim, é de se esperar que P2 apresente elevada riqueza, uma vez que eventos catastróficos possam ser compensados pela plasticidade no desenvolvimento larval aliado ao fluxo gênico entre as metapopulações existentes entre os diferentes corpos d'água desta localidade.

Em contraste, a menor riqueza de espécies registrada no sítio P1 (córrego no interior de floresta de galeria) pode estar relacionada aos modos reprodutivos das espécies registradas. Segundo Duellman \& Trueb (1986) em habitat sazonais com uma estação seca, o modo generalizado (onde os ovos são depositados em corpos de água lênticos e com girinos aquáticos) é mais frequente, como evidenciado por Hoogmoed \& Gorzula (1979) em região savânica na Venezuela, em que $57,7 \%$ das espécies apresentam o modo generalizado, no Cerrado, 52\% das espécies (Kopp et al. 2010), no Chaco Argentino, $50 \%$ das espécies (Perotti 1997) e no Pantanal, 62,5\% das espécies (Prado et al. 2005) e no presente estudo, $47 \%$ espécies apresentam modo reprodutivo generalizado.

Considerando que os corpos d'água estudados estão inseridos em área de intensa agricultura sofrendo elevado grau de perturbação antrópica, esses ambientes apresentam elevada riqueza de espécies, constituindo-se como importantes refúgios para anurofauna da região. Entretanto, as espécies registradas, de forma geral, são associadas a áreas antropizadas ou fitofisionomias abertas sendo favorecidas com a criação de ambientes artificiais como os observados neste estudo.

\section{Agradecimentos}

Os autores agradecem os proprietários e funcionários da granja Ziane pelo apoio ao estudo; Christine Strüssmann pelas sugestões na versão preliminar do manuscrito, Rogério J. Custódio pelo auxílio nas coletas em campo, Thamy S. Ribeiro e Mariana F. Felismino pelas correções do texto em inglês, e a Coordenação de Aperfeiçoamento de Pessoal de Nível Superior (CAPES) pelo suporte financeiro a F.H. Oda.

\section{Referências Bibliográficas}

BARRETO, L. \& MOREIRA, G. 1996. Seasonal variation in age structure and spatial distribution of a savanna larval anuran assemblage in Central Brazil. J. Herpetol. 30:87-92. http://dx.doi.org/10.2307/1564716

BERNARDE, P.S. \& KOKUBUM, M.N.C. 1999. Anurofauna do município de Guararapes, Estado de São Paulo, Brasil (Amphibia, Anura). Acta Biol. Leopoldensia 21(1):89-97.

BERVEN, K.A. 1981. Mate choice in the wood frog, Rana sylvatica. Evolution 35:707-722. http://dx.doi.org/10.2307/2408242

BERVEN, K.A. 1982. The genetic basis of altitudinal variation in the wood frog, Rana sylvatica. I. An experimental analysis of life history traits. Evolution 36:962-983. http://dx.doi.org/10.2307/2408075

BERVEN, K.A. 1990. Factors affecting population fluctuations in larval and adult stages of the wood frog (Rana sylvatica). Ecology 71:1599-1608. http://dx.doi.org/10.2307/1938295

BERVEN, K.A. \& GILL, D.E. 1983. Interpreting geographic variation in life-history traits. Am. Zool. 23:85-97.

BRANDÃO, R.A. \& ARAÚJO, A.F.B. 2001. A herpetofauna associada às matas de galeria do Distrito Federal. In Cerrado: Caracterização e recuperação de Matas de Galeria (J.F. Ribeiro, C.E.L. Fonseca \& J.C. Sousa-Silva, eds.). Embrapa Cerrados, Planaltina, p.561-604.

BRASIL. 1982. Ministério das Minas e Energia. Departamento Nacional da Produção Mineral. Projeto RADAMBRASIL. Folha SD.21 Cuiabá: geologia, geomorfologia, pedologia, vegetação, uso potencial da terra. Rio de Janeiro, 544p.

BURNHAM, K.P. \& OVERTON, W.S. 1979. Robust estimation of population size when capture probabilities vary amoung animals. Ecology 60:927-936. http://dx.doi.org/10.2307/1936861

CARDOSO, A.J., ANDRADE, G.V. \& HADDAD, C.F.B. 1989. Distribuição espacial em comunidades de anfíbios (Anura) no sudeste do Brasil. Rev. Bras. Biol. 49(1):241-249.

CAVALCANTI, R.B. \& JOLY, C.A. 2002. Biodiversity and conservation priorities in the Cerrado region. In The Cerrados of Brazil: Ecology and Natural History of a Neotropical Savanna (P.S. Oliveira \& R.J. Marquis, eds.). Columbia University Press, New York, p.351-367. 
COLLI, G.R., BASTOS, R.P., ARAUJO, A.F.B. 2002. The character and dynamics of the Cerrado herpetofauna. In The Cerrados of Brazil: Ecology and natural history of neotropical savanna (P.S. Oliveira \& R.J. Marquis, eds). New York: Columbia Univ. Press, p.223-241.

CONTE, C.E. \& MACHADO, R.A. 2005. Riqueza de espécies e distribuição espacial e temporal em comunidade de anfíbios anuros (Amphibia, Anura) em uma localidade do Município de Tijucas do Sul, Paraná, Brasil. Rev. Bras. Zool. 22(4):940-948. http://dx.doi.org/10.1590/S010181752005000400021

COLWELL, R.K., MAO, C.X. \& CHANG, J. 2004. Interpolating, extrapolating, and comparing incidence-based species accumulation curves. Ecology 85:2717-2727. http://dx.doi.org/10.1890/03-0557

COLWELL, R.K. 2005. EstimateS: Statistical estimation of species richness and shared species from samples. version 7.5. http://purl.oclc.org/ estimates (último acesso em 21/05/2012).

COURTEMANCH, D.L. 1996. Commentary on the sub-sampling procedures used for rapid bioassessments. J. N. Am. Benthol. Soc. 15(3):381-385. http://dx.doi.org/10.2307/1467284

CRUMP, M. \& SCOTT JUNIOR, N.J. 1994. Standard techniques for Inventory and Monitoring. In Measuring and Monitoring Biological Diversity (W.R. Heyer, M.A. Donnelly, R.W. McDiarmid, L.A.C. Hayek \& M.S. Foster, eds.). Smithsonian Institutions Press, Washington and London, p.84-92.

CRUZ, J.P., LEITE, H.G., SOARES, C.P.B., CAMPOS, J.C.C., SMIT, L. \& NOGUEIRA, G.S. 2008. Curvas de crescimento e de índice de local para povoamentos de Tectona grandis em Tangará da serra, Mato Grosso. Rev. Árvore. 31:669-676.

DUELLMAN, W.E. \& TRUEB, L. 1986. Biology of Amphibians. New York, McGraw-Hill.

DUELLMAN, W.E. \& TRUEB, L. 1994. Biology of Amphibians. The Johns Hopkins University Press, Baltimore.

DUFRÊNE, M. \& LEGENDRE, P. 1997. Species assemblages and indicator species: the need for flexible asymmetrical approach. Ecol. Monogr. 67(3):345-366.

ETEROVICK, P.C. \& SAZIMA, I. 2000. Structure of an anuran community in a montane meadow in southeastern Brazil: effects of seasonality, habitat, and predation. Amphibia-Reptilia. 21:439-461. http://dx.doi. org/10.1163/156853800300059331

GAUCH, H.G.J. 1982. Multivariate analysis in community ecology. Cambridge University Press, Cambridge, 298p. http://dx.doi.org/10.1017/ CBO9780511623332

GIARETTA, A.A., MENIN, M., FACURE, K.G., KOKUBUM, M.N.C. \& OLIVEIRA-FILHO, J.C. 2008. Species richness, relative abundance, and habitat of reproduction of terrestrial frogs in the Triângulo Mineiro region, Cerrado biome, southeastern Brazil. Iheringia, Sér. Zool. 98(2):181-188.

GOTELLI, N. \& COLWELL, R.K. 2001. Quantifying biodiversity: Procedures and pitfalls in the measurement and comparison of species richness. Ecol. Lett. 4:379-391. http://dx.doi.org/10.1046/j.1461-0248.2001.00230.x

GRIFFITHS, R.A. 1997. Temporary ponds as amphibian habitats. Aquat. Conserv.: Mar. Freshwat. Ecosyst. 7:119-126. http://dx.doi.org/10.1002/ (SICI)1099-0755(199706)7:2\%3C119::AID-AQC223\%3E3.0.CO;2-4

HAZELL, D., CUNNINGHAM, D.L., MACKEY, B. \& OSBORNE, W. 2001. Use of farm dams as frog habitat in an Australian agricultural landscape: factors affecting species richness and distribution. Biol. Conserv. 102:155-169. http://dx.doi.org/10.1016/S0006-3207(01)00096-9

HERRMANN, H.L., BABBITT, K.J., BABER, M.J. \& CONGALTON, R.G. 2005. Effects of landscape characteristics on amphibian distribution in a forest-dominated landscape. Biol. Conserv. 123:139-149. http:// dx.doi.org/10.1016/j.biocon.2004.05.025

HILL, M.O. \& GAUCH JUNIOR, H.G. 1980. Detrended Correspondence analysis: an improved ordination technique. Vegetation 42:47-58. http:// dx.doi.org/10.1007/BF00048870
HOOGMOED, M. \& GORZULA, S. 1979. Checklist of the savanna inhabiting frogs of the El Manteco region with notes on their ecology and the description of a new species of tree frog (Hylidae, Anura). Leiden, Zool. Meded. 54:183-216.

HOWARD, R.D. 1978. The evolution of mating strategies in bullfrogs, Rana catesbeiana. Evolution 32:850-871. http://dx.doi.org/10.2307/2407499

KNUTSON, M.G., SAUER, J.R., OLSEN, D.A., MOSSMAN, M.J., HEMESATH, L.M. \& LANNOO, M.J. 1999. Effects of landscape composition and wetland fragmentation on frog and toad abundance and species richness in Iowa and Wisconsin, U.S.A. Conserv. Biol. 13(6):1437-1446. http://dx.doi.org/10.1046/j.15231739.1999.98445.x

KOPP, K. \& ETEROVICK, P. 2006. Factors influencing spatial and temporal structure of frog assemblages at ponds in southeastern Brazil. J. Nat. Hist. 40:1813-1830. http://dx.doi.org/10.1080/00222930601017403

KOPP, K., SIGNORELLI, L. \& BASTOS, R.P. 2010. Distribuição temporal e diversidade de modos reprodutivos de anfíbios anuros no Parque Nacional das Emas e entorno, estado de Goiás, Brasil. Iheringia. Sér. Zool.100:192-200. http://dx.doi.org/10.1590/S0073-47212010000300002

LEIPS, J., McMANUS, M.G. \& TRAVIS, J. 2000. Response of Treefrog Larvae To Drying Ponds: Comparing Temporary and Permanent Pond Breeders. Ecology 81:2997-3008. http://dx.doi.org/10.1890/00129658(2000)081[2997:ROTLTD]2.0.CO;2

MELO, A.S. \& HEPP, L.U. 2008. Ferramentas estatísticas para análise de dados provenientes de biomonitoramento. Oecol. Bras. 12(3):463-486.

MORAIS, A.R., SIGNORELli, L., GAMBAle, P.G., KOPP, K., NOMURA, F., GUIMARÃES, L.D., VAZ-SILVA, W., RAMOS, J. \& BASTOS, R.P. 2011. Anfíbios anuros associados a corpos d'água do sudoeste do estado de Goiás (Brasil). Biota Neotrop. 11(3): http://www. biotaneotropica.org.br/v11n3/pt/abstract?inventory+bn03611032011 (último acesso em 20/05/2012).

NOGUEIRA, C., COLLI, G.R. \& MARTINS, M. 2009. Local richness and distribution of the lizard fauna in natural habitat mosaics of the Brazilian Cerrado. Austral Ecol. 34:83-96. http://dx.doi.org/10.1111/j.14429993.2008.01887.x

ODA, F.H., BASTOS, R.P. \& SÁ LIMA, M.A.D.C. 2009. Taxocenose de anfíbios anuros no Cerrado do Alto Tocantins, Niquelândia, Estado de Goiás: diversidade, distribuição local e sazonalidade. Biota Neotrop. 9(4): http://www.biotaneotropica.org.br/v9n4/en/abstract?inventory+ bn03609042009 (último acesso em 19/05/2012).

OLDEN, J.D., POFF, N.L., DOUGLAS, M.R, DOUGLAS, M.E \& FAUSCH, K.D. 2004. Ecological and evolutionary consequences of biotic homogenization. Trends Ecol. Evol. 19:18-24. 10.1016/j. tree.2003.09.010. http://dx.doi.org/10.1016/j.tree.2003.09.010

OLIVEIRA-FILHO, A.T. \& RATTER, J.A. 2002. Vegetation physiognomies and woody flora of the Cerrado Biome. In The Cerrados of Brazil: Ecology and Natural History of a Neotropical Savanna (P.S. Oliveira \& R.J. Marquis, eds.). Columbia University Press, Irvington.

PEROTTI, M.G. 1997. Reproductive modes and quantitative reproductive parameters of an anuran assemblage from the semiarid Chaco, Salta, Argentina. Rev. Chil. Hist. Nat. 70:277-288.

POMBAL JR, J.P. 1997. Distribuição espacial e temporal de anuros (Amphibia) em uma poça permanente na Serra de Paranapiacaba, sudeste do Brasil. Rev. Bras. Biol. 57(4):583-594.

POUGH, F.H. \& KAMEL, S. 1984. Post-metamorphic change in activity metabolism of anurans in relation to life history. Oecologia. 65:138-144. http://dx.doi.org/10.1007/BF00384476

PRADO, C.P.A., UETANABARO, M. \& HADDAD, C.F.B. 2005. Breeding activity patterns, reproductive modes, and habitat use by anurans (Amphibia) in a seasonal environment in the Pantanal, Brasil. AmphibiaReptilia 26:211-221. http://dx.doi.org/10.1163/1568538054253375 
SANTOS, A.J. 2004. Estimativa de riqueza em espécies. In Métodos de Estudo em Biologia da Conservação e Manejo da Vida Silvestre (L. Cullen, R. Rudran \& C Valladares-Padua, orgs.). Editora UFPR, Curitiba, p.19-41.

SANTOS, A.J. 2006. Estimativas de riqueza em espécies. In Métodos de estudo em Biologia da Conservação \& Manejo da Vida Silvestre (L. Cullen, R. Rudran, \& C. Valladares-Padua, Eds.). Editora da Universidade Federal do Paraná, Curitiba, p.19-43.

SANTOS, T.G., ROSSA-FERES, D.C. \& CASATTI, L. 2007. Diversidade e distribuição espaço-temporal de anuros em região com pronunciada estação seca do sudeste do Brasil. Iheringia, Sér. Zool. 97(1):37-49.

SANTOS, T.G., VASCONCELOS, T.S., ROSSA-FERES, D.C. \& HADDAD, C.F.B. 2009. Anurans of a seasonally dry tropical forest: Morro do Diabo State Park, São Paulo state, Brazil. J. Nat. Hist. 43:973-993. http://dx.doi. org $/ 10.1080 / 00222930802702498$

SEMLITSCH, R.D \& WILBUR, H.M. 1988. Effects of pond drying time on metamorphosis and survival in the salamander Ambystoma talpoideum. Copeia 1988:978-983. http://dx.doi.org/10.2307/1445721

SKELLY, D.K. 1997. Pond permanence and predation are powerful forces shaping the structure of tadpole communities. Tadpole communities. Am. Sci. 85:36-45.

SMITH, D.C. 1983. Factors controlling tadpole populations of the chorus frog (Pseudacris triseriata) on Isle Royale, Michigan. Ecology 64(3):501-510. http://dx.doi.org/10.2307/1939970

SMITH, D.C. 1987. Adult recruitment in chorus frogs: effects of size and date at metamorphosis. Ecology 68:344-350. http://dx.doi. org $/ 10.2307 / 1939265$

SOARES-FILHO, B.S., NEPSTAD, D., CURRAN, L., VOLL, E., CERQUEIRA, G., GARCIA, R.A., RAMOS, C.A., MCDONALD, A., LEFEBVRE, P. \& SCHLESINGER, P. 2006. Modeling conservation in the Amazon basin. Nature 440:520-523. http://dx.doi.org/10.1038/ nature 04389
SOARES, J.L. \& NOGUEIRA, A.C.R. 2008. Depósitos carbonáticos de Tangará da Serra-MT: Uma nova ocorrência de capa carbonática neoproterozóica no sul do Cráton Amazônico. Rev. Bras. Geoc. 38:722-739.

TER BRAAK, C.J.F. 1995. Ordination. In Data analysis in community and landscape ecology (R.H.G. Jongman, C.J.F. Ter Braak \& O.F.R. Van Tongeren, eds.). Cambridge University Press, Cambrigde, p.91-173.

VASCONCELOS, T.S. \& ROSSA-FERES, D.C. 2005. Diversidade, distribuição espacial e temporal de anfíbios anuros (Amphibia, Anura) na região noroeste do estado de São Paulo, Brasil. Biota Neotrop. 5(2): http:// www.biotaneotropica.org.br/v5n2/pt/abstract?article+BN01705022005 (último acesso em 19/05/2012).

VASCONCELOS, T.S., SANTOS, T.G., ROSSA-FERES, D.C. \& HADDAD, C.F.B. 2009. Influence of the environmental heterogeneity of breeding ponds on anuran assemblages from southeastern Brazil. Can. J. Zoolog. 87:699-707. http://dx.doi.org/10.1139/Z09-058

WELLS, K.D. 2007. The ecology and behavior of amphibians. University of Chicago Press, Chicago. http://dx.doi.org/10.7208/ chicago/9780226893334.001.0001

WILLIAMS, P., WHITFIELD, M., BIGGS, J., BRAY, S., FOX, G., NICOLET, P. \& SEAR, D. 2003. Comparative biodiversity of rivers, streams, ditches and ponds in an agricultural landscape in Southern England. Biol. Conserv. 115:329-341. http://dx.doi.org/10.1016/S00063207(03)00153-8

WOODWARD, B.D. 1983. Predator-prey interactions and breeding pond use of temporary pond species in a desert anuran community. Ecology 64:1549-1555. http://dx.doi.org/10.2307/1937509

ZAR, J.H. 1999. Biostatistical analysis. New Jersey, Prentice Hall, 929p.

ZIMMERMAN, B.L. 1994. Audio strip transects. In Measuring and Monitoring Biological Diversity (W.R. Heyer, M.A. Donnelly, R.W. McDiarmid, L.A.C. Hayek \& M.S. Foster, eds.). Smithsonian Institutions Press, Washington and London, p.92-97.

Recebido em 24/07/2012

Versão reformulada recebida em 12/12/2012

Publicado em 12/03/2013 